\section{Aquaporin water channels: atomic structure and molecular dynamics meet clinical medicine}

\author{
David Kozono, ${ }^{1}$ Masato Yasui, ${ }^{1,2}$ \\ Landon S. King, ${ }^{1,3,4}$ and Peter Agre ${ }^{1,3}$ \\ ${ }^{1}$ Department of Biological Chemistry; \\ ${ }^{2}$ Department of Pediatrics, Division of Neonatology; \\ ${ }^{3}$ Department of Medicine; and \\ ${ }^{4}$ Department of Medicine, Division of Pulmonary and Critical \\ Care Medicine; Johns Hopkins University School of Medicine, \\ Baltimore, Maryland, USA
}

Address correspondence to: Peter Agre, Johns Hopkins University School of Medicine, 725 North Wolfe Street, Baltimore, Maryland 21205, USA. Phone: (410) 955-3154;

Fax: (410) 955-7049; E-mail: pagre@jhmi.edu.

J. Clin. Invest. 109:1395-1399 (2002). doi:10.1172/JCI200215851.

The movement of water across cell membranes is fundamental to life. Water constitutes roughly $70 \%$ of the mass of most living organisms, so the orderly distribution of water is required to maintain proper fluid balance within different anatomic compartments. Although water is known to diffuse through lipid bilayers, diffusion is not sufficiently rapid for many physiological processes. To accommodate these needs, a family of membrane channel proteins evolved for rapid transport of water across biological membranes. These proteins, termed "aquaporins," are found in all life forms, including archaea, eubacteria, fungi, plants, and all phyla of animals.

Since the discovery of the aquaporins ten years ago (1), researchers around the world have sought to learn how these proteins work. Selectivity, rates of permeation, and gating mechanisms are three properties that characterize channel proteins. Aquaporins are exquisitely selective for the transport of water even repelling hydronium ions $\left(\mathrm{H}_{3} \mathrm{O}^{+}\right)$. The importance of the latter is emphasized by the normal function of mammalian renal tubules, which reabsorb $99 \%$ of the water from glomerular filtrate at the same time that acid is secreted by intercalated cells. Some members of the aquaporin superfamily are permeated by a variety of small neutral solutes such as glycerol or urea (termed "aquaglyceroporins") (2). The transport of water or glycerol through aquaporins represents facilitated diffusion driven by osmotic or concentration gradients.

\section{Atomic structures}

Early studies of molecular recombinants predicted the "hourglass" model for aquaporin structure (3). Each
$28-\mathrm{kDa}$ subunit in the aquaporin homotetramer contains an individual aqueous pore. The primary sequence of aquaporin-1 (AQP1) reveals two tandem sequence repeats, each encoding three transmembrane helices with a short loop (hemipore) connecting the second and third helices. The hemipore loops enter but do not span the bilayer, and each contains the signature motif Asn-Pro-Ala (NPA). The amino- and carboxy-termini are intracellular, so the repeats are oriented at $180^{\circ}$ to each other. The two hemipores fold into the membrane from the opposite surfaces of the bilayer, overlapping midway through the bilayer where they are surrounded by six transmembrane helices.

Several recent studies have revealed the high-resolution structures of the water channel protein family that explain their selectivity. The structure of human AQP1 reconstituted into membrane crystals was determined at $3.8 \AA$ A by cryoelectron microscopy $(4,5)$; the structure of three-dimensional crystals of the Escherichia coli aquaglyceroporin (GlpF) was obtained at $2.2 \AA$ by $x$-ray diffraction analysis (6). Remarkably similar, AQP1 and GlpF were referred to as "fraternal twins" (7). By merging these coordinates, a refined structure for $\mathrm{AQP} 1$ was obtained (Figure 1) (8). Importantly, the atomic structure of AQP1 was also recently determined by x-ray analysis of bovine AQP1 crystals (9). Understanding the atomic structure of both AQP1 and GlpF has permitted molecular dynamics calculations to simulate their behavior during membrane water and glycerol transport (10-12). Together these investigations have provided remarkably uniform agreement of structure with profound insight into the functions of these proteins.

\section{Membrane transport of $\mathrm{H}_{2} \mathrm{O}$ but not $\mathrm{H}_{3} \mathrm{O}^{+}$}

The structure of AQP1 provides a uniquely selective mechanism for free permeation by water through a channel with no moving parts (Figure 2). Glycerol permeation of aquaglyceroporins is similar. Theoretically, protons could traverse a channel by transiently interacting with successive hydrogen-bonded water molecules in a stationary, single-file chain (analogous to a bucket brigade or an electrical wire). This model of proton conductance has been demonstrated through the column of water molecules in gramicidin A (13). Two major features of the AQP1 channel prevent the formation of a continuous chain of hydrogen-bonded water molecules (Figure 2).

The first barrier to proton permeation is located at the narrowest constriction of the aqueous pathway through AQP1. Approximately $8 \AA$ above the middle of the bilayer, the channel is lined by the side chains of Arg-195, Phe-56, and His-180 (represented in sagittal section in Figure 2). The backbone carbonyl atoms of Gly-188 and Cys-189 also line the channel at this level, and the 2.8- $\AA$ diameter of the pore approximates the mean van der 


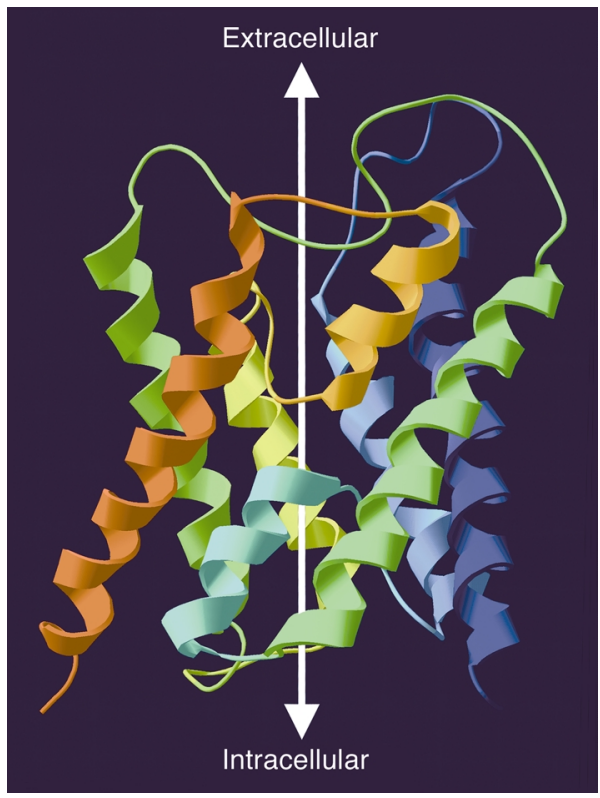

Waals diameter of a water molecule (represented in transverse section in Figure 3). The residue at the constriction Arg-195 is conserved in virtually all members of the aquaporin superfamily and bears a strong positive charge serving to repel protonated water $\left(\mathrm{H}_{3} \mathrm{O}^{+}\right)$. Molecular dynamics simulations revealed that hydrogen-bonded water chains are interrupted adjacent to Arg-195 (10). The pore-lining residue His-180 is conserved in all water-selective mammalian aquaporins and restricts the diameter of the pore with a partial positive charge at neutral $\mathrm{pH}$. In contrast, multifunctional aquaporins that have alternate residues at this position have larger diameters. GlpF, with a glycine residue in the position analogous to His-180, has a pore diameter approximately $1 \AA$ Å wider, permitting lengthwise passage of polyols such as glycerol (6).

The second barrier to proton permeation is the strong dipole, formed by the two short pore helices

\section{Figure 2}

Schematic architecture of the channel within an AQP1 subunit (sagittal section). The shape of the aqueous pore (blue) is derived from calculations based on the structure of bovine AQP1 (9). Four water molecules shown in bold colors represent transient interactions with the pore-lining residues at discrete sites. Bulk water in the extracellular and intracellular vestibules is depicted in pastels. Three features of the channel specify selectivity for water: (a) Size restriction. Eight angstroms above the midpoint of the channel, the pore narrows to a diameter of $2.8 \AA$ (approximately the diameter of a water molecule). (b) Electrostatic repulsion. A conserved residue (Arg-195) at the narrowest constriction of the pore imposes a barrier to cations, including protonated water $\left(\mathrm{H}_{3} \mathrm{O}^{+}\right)$. (c) Water dipole reorientation. Two partial helices meet at the midpoint of the channel, providing positively charged dipoles that reorient a water molecule as it traverses this point. Disrupting hydrogen bonding in the single-file chain water molecules prevents the formation of a proton conductance. A video animation of water molecules passing through the AQP1 protein is available on the internet at http://www.mpibpc.gwdg.de/abteilungen/071/bgroot/presentations/aqp1_dyn/aqp1_mono.html.

\section{Figure 1}

Ribbon diagram of the structure of an AQP1 subunit (sagittal section). The model is based on Protein Data Bank coordinates $1 \mathrm{H} 6 \mathrm{I}$ of human red cell AQP1 (8). The amino-terminus is blue and the carboxy-terminus is red. Six membrane-spanning helices surround two hemipores (loops with short helices - cyan and orange) that meet in the center of the bilayer. The white arrow illustrates the aqueous channel through the protein.

containing the motif NPA in the hemipore loops that meet at the center of AQP1 (Figure 2). Importantly, these partial charges and Asn residues reorient water molecules passing through the channel $(4,14)$. This water dipole reorientation disrupts hydrogen-bonding interactions between this water molecule and those both above and beneath it, eliminating the possibility of proton conductance.

With very few exceptions, ions are not known to permeate aquaporins. Theoretically, dehydrated ions could fit through a 2.8 -Å-diameter pore. The dehydrated sodium ion has a diameter of $1.9 \AA$; however, the diameter of the hydrated sodium ion is $7.16 \AA$ (15). Cation channels with narrow pores possess mechanisms to dehydrate ions. For example, one side of the KcsA potassium channel pore contains 16 carbonyl oxygen atoms arranged in a stacked ring configuration. This configuration is sufficient to completely substitute for the interactions with waters of hydration (16). In contrast, AQP1 contains four carbonyl oxygen atoms on each side of the pore. Although this arrangement can efficiently isolate water molecules from bulk water, it can only partially dehydrate ions, which remain too large to pass through the narrowest constriction of the water channel.

\section{Ultra-high-speed transport}

The crystallographic and dynamic structures of AQP1 demonstrate features of the water channel that facilitate rapid water transport. A single column of water molecules resides in a $20-\AA$ span within the channel.

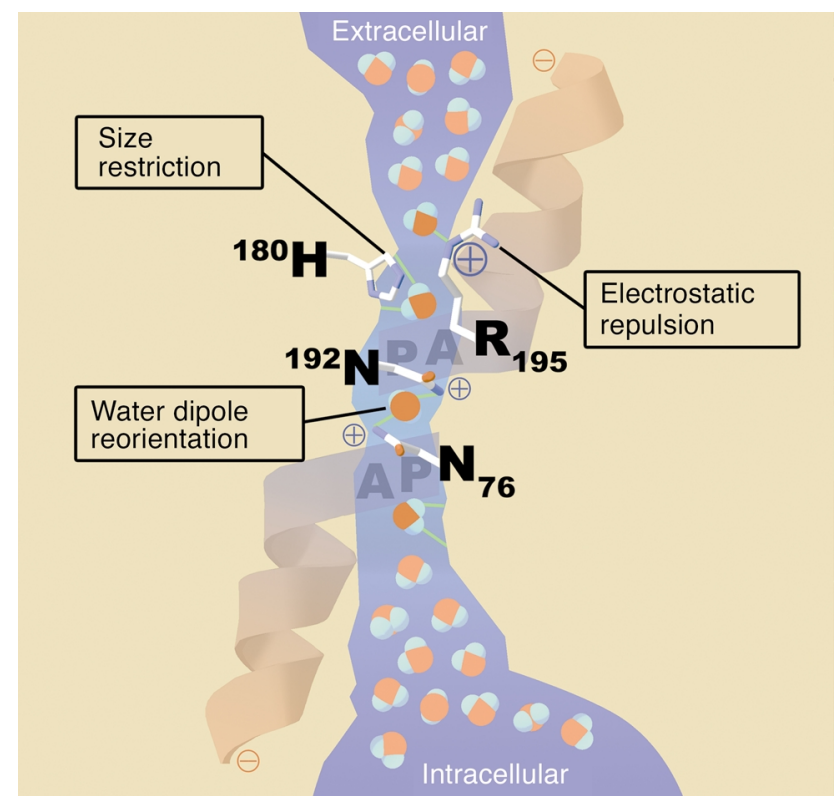


Discrete sites of interaction between water molecules and pore lining residues are predicted (Figure 2); the remainder of the channel is largely hydrophobic. Water molecules jump from site to site in a manner analogous to saltatory conduction at the nodes of Ranvier in myelinated axons. The rate of water transport through AQP1 (approximately $3 \times 10^{9}$ water molecules per subunit per second) (17) is considerably faster than that of other described channels; potassium permeates at a rate of $1 \times 10^{8}$ ions per second through the KcsA channel, which is among the fastest ion channels (18). Permeation would be greatly decreased if water molecules interacted with other polar sites lining the pore. A dramatic video animation of water molecules passing through the AQP1 protein is available at the website indicated in the legend to Figure 2.

Gating is physiologically vital for many channel proteins. Most described ion channels have well-established gating mechanisms, such as the ball and chain system of the Drosophila Shaker channels (19), or the regulation of ligand-gated NMDA receptors by a poreblocking magnesium ion (20). Although it is not a general feature of aquaporins, a subset of aquaporins may be gated. The major intrinsic protein of bovine lens (MIP, AQP0) is activated by low $\mathrm{pH}$ and inactivated by calcium ions (21); AQP3 is inactivated by low $\mathrm{pH}(22)$; and AQP6 in intracellular vesicles within $\alpha$-intercalated cells of the renal collecting duct is activated by low $\mathrm{pH}$ (23). In addition, gating of the yeast aquaglyceroporin FPS1 has been shown to involve an $\mathrm{N}$-terminal domain (24). The recent structural biology reports do not reveal mechanisms for gating of aquaporins, although molecular dynamics simulations suggest that the water permeability of the aquaglyceroporin $\mathrm{GlpF}$ decreases upon removal of glycerol from the environment - an example of induced-fit gating where the permeant solute induces its own permeability and that of other solutes (10).

\section{Aquaporins and human clinical disorders}

The atomic structure of AQP1 protein provides marked insight into several human disease states. Ten other aquaporin genes are also expressed in humans, and their structures are expected to be very similar. Each aquaporin is present in specific tissues where their permeabilities to water and small solutes may contribute to multiple physiological processes. So far, investigators have identified mutations in the water-selective human homologs AQP0, AQP1, and AQP2.

AQP0 is the major intrinsic protein of lens fiber cells. Unlike the majority of water-selective aquaporins, the permeability of AQP0 is relatively low, conferring a modest four- to fivefold increase in water permeability over the intrinsic permeability of lipid bilayers (25). The physiological importance of this protein may lie in facilitating cell-to-cell adherence between lens fiber cells via interactions between AQP0 molecules in adjacent cells (26). The extracellular contours of the protein create tongue-and-groove contacts that allow the tight fit of juxtaposed AQP0 molecules. Disruption of these contacts may interfere with uniformity and transparency of the lens.
Two kindreds with dominantly inherited congenital cataracts were recently identified with AQP0 mutations: Glu-134 $\rightarrow$ Gly or Thr-138 $\rightarrow$ Arg (27). Glu-134 in AQP0 corresponds to Glu-142 in AQP1 and is conserved among all aquaporins. The negatively charged carboxyl side chain is critical for alignment of the backbone carbonyl atoms that isolate a water molecule entering from the extracellular side of the channel (Figure 3). The Thr-138 $\rightarrow$ Arg mutation may perturb the proper orientation of Glu-134 by introducing an adjacent positive charge. Both mutations interrupt trafficking of nascent polypeptide to the plasma membrane (28). Although the sites of the mutations lie along the same face of the fourth transmembrane helix, the phenotypes of the cataracts are, curiously, distinct in these families. Patients with the Glu-134 $\rightarrow$ Gly mutation have single lamellar opacities, whereas those with the Thr-138 $\rightarrow$ Arg mutation have multifocal opacities throughout the lens (28).

AQP1 is expressed in red cells, renal proximal tubules, capillary endothelium, and multiple other sites. Patients with inherited mutations in the gene for AQP1 fail to express the protein and were identified because they lack the Co blood group antigen (29), determined by a polymorphism in the first extracellular domain

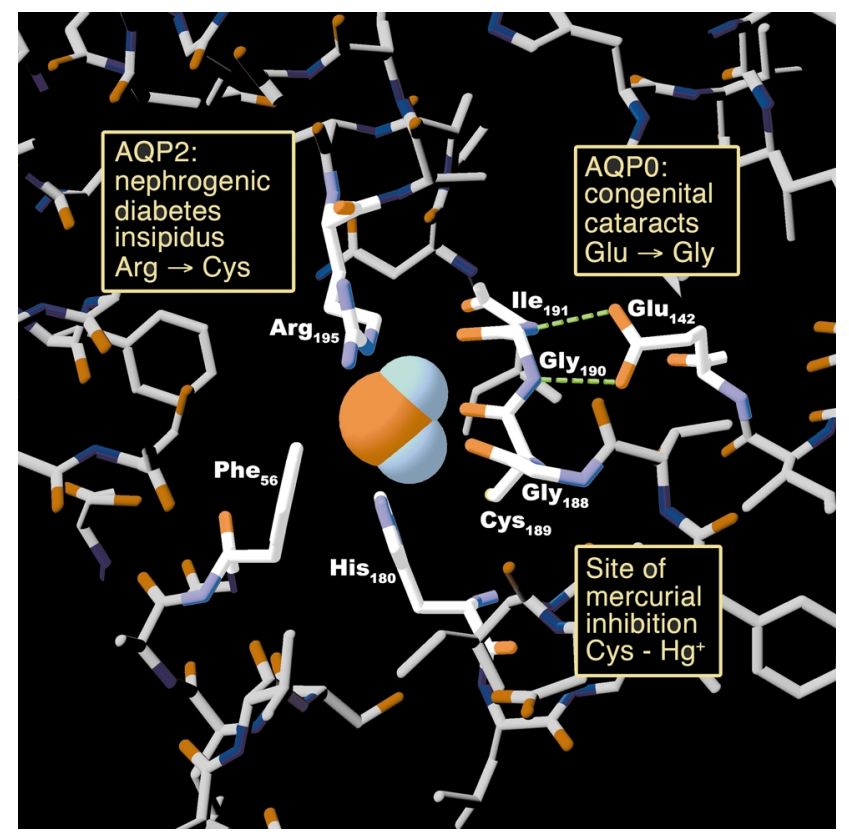

Figure 3

Human disease mutations and pharmacologically active sites projected onto an atomic model of an AQP1 pore at the narrowest constriction point (transverse section). The model is based on Protein Data Bank coordinates $1 \mathrm{H} 6 \mathrm{I}$ of human red cell AQP1 (8). The side chains of three residues (Arg-195, His-180, and Phe-56) plus the backbone carbonyl groups of two residues (Gly-188 and Cys-189) line the pore. Mutation in AQP2 of the residue corresponding to Arg-195 results in autosomal recessive nephrogenic diabetes insipidus. The side chain of Cys-189 lies proximal to the pore. Binding of a mercuric ion to the sulfhydryl group of this residue inhibits the water permeability of AQP1. Hydrogen bonding between Glu-142 and the backbone amide groups of Gly-190 and Ile-191 orients the carbonyl groups of these residues toward the pore. Mutation in AQP0 of the residue corresponding to Glu-142 results in dominantly inherited congenital cataracts. 
(30). Except for their unique blood transfusion requirements, these AQP1-null individuals are not symptomatic in the unstressed state, although careful clinical testing shows that they have renal concentration defects (31) and reduced water permeability in the peribronchiolar capillary plexus (32). Either of these abnormalities could be of great clinical significance if the individuals should be deprived of water or sustain fluid overload from any cause.

AQP2 is predominantly expressed in the principal cells in the collecting ducts of renal tubules where regulated water reuptake is known to occur. In the diuretic state, AQP2 resides in intracellular vesicles. Upon stimulation by vasopressin (antidiuretic hormone), the vesicles traffic to the plasma membrane, increasing the water permeability of the collecting duct (33). Nephrogenic diabetes insipidus (NDI) patients suffer from defective urinary concentration resulting from kidney resistance to vasopressin. Patients with X-linked NDI carry mutations in the gene encoding the vasopressin $\mathrm{V} 2$ receptor, whereas patients with recessively inherited NDI have mutations in the $A Q P 2$ gene, affecting the water transporting part of the protein $(34,35)$. One mutation localizes to Arg-187 (corresponding to Arg195 residue in AQP1), which disrupts the normal folding of the narrow constriction in the aqueous pore (Figure 3). Patients with dominantly inherited NDI have a mutation in the carboxy-terminus near the site that regulates exocytosis (36). Unlike patients with $A Q P 1$ mutations, $A Q P 2$ homozygotes and compound heterozygotes are severely affected clinically. Altered expression levels of AQP2 have been identified in most known defects of urine concentration or fluid retention. For example, AQP2 is underexpressed in diabetes insipidus and nocturnal enuresis, and AQP2 is overexpressed in congestive heart failure and pregnancy $(37,38)$.

With recent advances in genome biology and highthroughput sequencing, it may become possible to rapidly identify mutations in all 11 human aquaporin genes. Analysis of mice with targeted gene disruptions of each of several different aquaporin genes is being reported, and each mutant has been shown to have a defect in fluid transport (39). Although it is unlikely that the mice and humans will have equivalent clinical phenotypes, it is certain that multiple human aquaporin defects will be identified. Insights gained by examining the structures of aquaporins can help distinguish disease-causing mutations from silent polymorphisms.

\section{Clinical pharmacology}

Knowledge of the architecture of aquaporins may also play a role in toxicology or pharmacology. Before the discovery of the water channels, scientists observed mercury-sensitive water transport in red blood cells (40). Biochemical studies revealed that the inhibition might be due to the interaction of mercury ions with Cys-189, predicted to reside within the pore of AQP1 $(3,41)$. Structural models of the protein confirm this prediction, and one can expect that covalent modification of the sulfhydryl residue may occlude the channel (Figure 3). Aquaporins may prove to be pharmacological targets. Agents that serve to block water channels may be useful in fluid overload states as diuretics. Of note, the agent calomel $\left(\mathrm{Hg}_{2} \mathrm{Cl}_{2}\right)$ was once used for refractory fluid overload. Agents that increase expression of the protein, either by induction of transcription (42) or by reduction of ubiquitination-dependent degradation (43), could be beneficial when dissipation of edema is desired. Each aquaporin would be expected to have organ-specific functions. Knowledge of aquaporin structure may facilitate rational drug design.

\section{Implications}

Aquaporins are suspected in numerous disorders involving fluid transport such as brain edema, cirrhosis, congestive heart failure, glaucoma, and pre-eclampsia. Multifunctional aquaglyceroporins that transport glycerol and other small molecules may have roles in energy metabolism and heavy metal transport (44), and at least one member of the aquaporin family may have a role in acid-base homeostasis as a kidney anion channel. Recent advances in determining the structures of aquaporins at the atomic level have revealed key mechanisms by which these channels maintain exquisite selectivity for substrates without sacrificing high rates of transport. Further investigation may reveal that structural signals lead to differences in several aspects of aquaporin function, such as the basis (and apparent requirement) for oligomerization, channel permeability properties, stability, and trafficking. Knowledge of protein structure may also provide insight into the spectrum of disease caused by distinct mutations in a single aquaporin gene, as in the case of mutations in $\mathrm{AQP} 0$ or AQP2. Finally, it is hoped that mechanistic and structural insights will lead to the development of new therapeutics through rational drug design. Considering the diverse expression patterns and functional properties of the aquaporins, the application of aquaporin structural biology holds promise for a wide range of clinical disorders.

\section{Acknowledgments}

This work was supported by grants from the NIH and the Human Frontier Science Program Organization.

1. Preston, G.M., Carroll, T.P., Guggino, W.B., and Agre, P. 1992. Appearance of water channels in Xenopus oocytes expressing red cell CHIP28 protein. Science. 256:385-387.

2. Borgnia, M., Nielsen, S., Engel, A., and Agre, P. 1999. Cellular and molecular biology of the aquaporin water channels. Annu. Rev. Biochem. 68:425-458.

3. Jung, J.S., Preston, G.M., Smith, B.L., Guggino, W.B., and Agre, P. 1994. Molecular structure of the water channel through aquaporin CHIP. The hourglass model. J. Biol. Chem. 269:14648-14654.

4. Murata, K., et al. 2000. Structural determinants of water permeation through aquaporin-1. Nature. 407:599-605.

5. Ren, G., Reddy, V.S., Cheng, A., Melnyk, P., and Mitra, A.K. 2001. Visualization of a water-selective pore by electron crystallography in vitreous ice. Proc. Natl. Acad. Sci. USA. 98:1398-1403.

6. Fu, D., et al. 2000. Structure of a glycerol-conducting channel and the basis for its selectivity. Science. 290:481-486.

7. Unger, V.M. 2000. Fraternal twins: AQP1 and GlpF. Nat. Struct. Biol. 7:1082-1084.

8. de Groot, B.L., Engel, A., and Grubmuller, H. 2001. A refined structure of human aquaporin-1. FEBS Lett. 504:206-211.

9. Sui, H., Han, B.G., Lee, J.K., Walian, P., and Jap, B.K. 2001. Structural basis of water-specific transport through the AQP1 water channel. Nature. 414:872-878.

10. de Groot, B.L., and Grubmuller, H. 2001. Water permeation across 
biological membranes: mechanism and dynamics of aquaporin-1 and GlpF. Science. 294:2353-2357.

11. Jensen, M.O., Tajkhorshid, E., and Schulten, K. 2001. The mechanism of glycerol conduction in aquaglyceroporins. Structure (Camb.) 9:1083-1093.

12. Kong, Y., and Ma, J. 2001. Dynamic mechanisms of the membrane wate channel aquaporin-1 (AQP1). Proc. Natl. Acad. Sci. USA. 98:14345-14349.

13. Pomes, R., and Roux, B. 1996. Structure and dynamics of a proton wire: a theoretical study of $\mathrm{H}+$ translocation along the single-file water chain in the gramicidin A channel. Biophys. J. 71:19-39.

14. Tajkhorshid, E., et al. 2002. Control of the selectivity of the aquaporin water channel family by global orientational tuning. Science. 296:525-530.

15. Nightingale, E.R. 1959. Phenomenological Theory of Ion Solvation-Effective Radii of Hydrated Ions. J. Phys. Chem. 63:1381-1387.

16. Doyle, D.A., et al. 1998. The structure of the potassium channel: molecular basis of $\mathrm{K}+$ conduction and selectivity. Science. 280:69-77.

17.Zeidel, M.L., Ambudkar, S.V., Smith, B.L., and Agre, P. 1992. Reconstitution of functional water channels in liposomes containing purified red cell CHIP28 protein. Biochemistry. 31:7436-7440.

18. Morais-Cabral, J.H., Zhou, Y., and MacKinnon, R. 2001. Energetic optimization of ion conduction rate by the $\mathrm{K}^{+}$selectivity filter. Nature 414:37-42.

19. Demo, S.D., and Yellen, G. 1991. The inactivation gate of the Shaker K+ channel behaves like an open-channel blocker. Neuron. 7:743-753.

20. Premkumar, L.S., and Auerbach, A. 1996. Identification of a high affinity divalent cation binding site near the entrance of the NMDA receptor channel. Neuron. 16:869-880.

21. Nemeth-Cahalan, K.L., and Hall, J.E. 2000. $\mathrm{pH}$ and calcium regulate the water permeability of aquaporin 0. J. Biol. Chem. 275:6777-6782.

22. Zeuthen, T., and Klaerke, D.A. 1999. Transport of water and glycerol in aquaporin 3 is gated by $\mathrm{H}(+)$. J. Biol. Chem. 274:21631-21636.

23. Yasui, M., et al. 1999. Rapid gating and anion permeability of an intracellular aquaporin. Nature. 402:184-187.

24. Tamas, M.J., et al. 1999. Fps1p controls the accumulation and release of the compatible solute glycerol in yeast osmoregulation. Mol. Microbiol. 31:1087-1104

25. Mulders, S.M., et al. 1995. Water channel properties of major intrinsic protein of lens. J. Biol. Chem. 270:9010-9016.

26. Fotiadis, D., et al. 2000. Surface tongue-and-groove contours on lens MIP facilitate cell-to-cell adherence. J. Mol. Biol. 300:779-789.

27. Berry, V., Francis, P., Kaushal, S., Moore, A., and Bhattacharya, S. 2000 Missense mutations in MIP underlie autosomal dominant 'polymorphic' and lamellar cataracts linked to 12q. Nat. Genet. 25:15-17.

28. Francis, P., et al. 2000. Functional impairment of lens aquaporin in two families with dominantly inherited cataracts. Hum. Mol. Genet. 9:2329-2334.
29. Preston, G.M., Smith, B.L., Zeidel, M.L., Moulds, J.J., and Agre, P. 1994. Mutations in aquaporin-1 in phenotypically normal humans without functional CHIP water channels. Science. 265:1585-1587.

30. Smith, B.L., Preston, G.M., Spring, F.A., Anstee, D.J., and Agre, P. 1994. Human red cell aquaporin CHIP. I. Molecular characterization of ABH and Colton blood group antigens. J. Clin. Invest. 94:1043-1049.

31. King, L.S., Choi, M., Fernandez, P.C., Cartron, J.P., and Agre, P. 2001. Defective urinary-concentrating ability due to a complete deficiency of aquaporin-1. N. Engl. J. Med. 345:175-179.

32. King, L.S., Nielsen, S., Agre, P., and Brown, R.H. 2002. Decreased pulmonary vascular permeability in aquaporin-1-null humans. Proc. Natl. Acad. Sci. USA. 99:1059-1063.

33. Nielsen, S., et al. 1995. Vasopressin increases water permeability of kidney collecting duct by inducing translocation of aquaporin-CD water channels to plasma membrane. Proc. Natl. Acad. Sci. USA. 92:1013-1017.

34. Deen, P.M., et al. 1994. Requirement of human renal water channel aquaporin-2 for vasopressin-dependent concentration of urine. Science. 264:92-95.

35. Van Lieburg, A.F., et al. 1994. Patients with autosomal nephrogenic diabetes insipidus homozygous for mutations in the aquaporin 2 waterchannel gene. Am. J. Hum. Genet. 55:648-652.

36. Mulders, S.M., et al. 1998. An aquaporin-2 water channel mutant which causes autosomal dominant nephrogenic diabetes insipidus is retained in the Golgi complex. J. Clin. Invest. 102:57-66.

37. Schrier, R.W., Ohara, M., Rogachev, B., Xu, L., and Knotek, M. 1998. Aquaporin- 2 water channels and vasopressin antagonists in edematous disorders. Mol. Genet. Metab. 65:255-263.

38. Morello, J.P., and Bichet, D.G. 2001. Nephrogenic diabetes insipidus. Annu. Rev. Physiol. 63:607-630.

39. Verkman, A.S. 2000. Physiological importance of aquaporins: lessons from knockout mice. Curr. Opin. Nephrol. Hypertens. 9:517-522.

40. Macey, R.I. 1984. Transport of water and urea in red blood cells. Am. J. Physiol. 246:C195-C203.

41. Preston, G.M., Jung, J.S., Guggino, W.B., and Agre, P. 1993. The mercurysensitive residue at cysteine 189 in the CHIP28 water channel. J. Biol. Chem. 268:17-20

42. Moon, C., King, L.S., and Agre, P. 1997. Aqp1 expression in erythroleukemia cells: genetic regulation of glucocorticoid and chemical induction. Am. J. Physiol. 273:C1562-C1570.

43. Leitch, V., Agre, P., and King, L.S. 2001. Altered ubiquitination and stability of aquaporin-1 in hypertonic stress. Proc. Natl. Acad. Sci. USA. 98:2894-2898.

44. Wysocki, R., et al. 2001. The glycerol channel Fps1p mediates the uptake of arsenite and antimonite in Saccharomyces cerevisiae. Mol. Microbiol. 40:1391-1401. 